\title{
Neurosurgery in the elderly patient
}

\section{Gabriel lacob*}

* University Hospital Bucharest, ROMANIA

\author{
Motto: \\ "Love and respect old age people because you are aging too" \\ Aparna Verma
}

\section{ABSTRACT}

Objective. For government officials and health providers, elderly population - aged 65 and over, especially neurosurgical patient, represent a larger concern, an increasing problem not only for socio-economic reasons related to the medical act, but also for additional care requirements which should be done by the family and society, including rehabilitation facilities, occupational \& physical therapy, speech therapists, visiting nurses, to insure an effective recuperation after hospital discharge. A retrospective study with 325 "elderly" patients cohort, aged 65 and over, admitted in the Neurosurgery Department undergoing common neurosurgical procedures, in the last five years offer an evaluation for neurosurgical procedures, outcomes, comorbidities, anaesthetic and analgesic requirements, outcome.

Material and method. This study was performed on patients aged 65 years or older, with neurosurgical diseases, admitted to the Neurosurgery or the Intensive Care Unit of our hospital, between 2014-2019. An analysis was made on variables such as age, pathology, comorbidities, length of hospital stay especially in the ICU unit, type of cranio-cerebral or spinal procedures performed, anaesthesia protocols, complications, performance status, re-admissions and mortality.

Results. Patients age were divided into three categories: between 65-70 years old there were 152 patients $(46,76 \%)$, between $70-85$ years old 93 patients $(28.61 \%)$ and over 85 years old 80 patients (24,61\%). 173 patients were females (53,23\%), 152 were males (46.76\%). The admission Glasgow Coma Scale (GCS) score to those over 85 years old was between 3-12 in 29 cases (8.02\%) with early death in 13 patients. Several comorbidities were noticed in 294 patients (90.15\%): cardiac, pulmonary, hematologic especially coumarinic overdose, hepatic and renal failures, psychiatric illnesses, concomitant systemic disease or immunosuppressed patients by decompensated diabetes, primitive cancers affecting various organs, infectious diseases, also severe osteoporosis, chronic ethylic intoxication, limiting surgical attitude, also obtaining the informed consent for surgery. There were $154(47,38 \%)$ patients with cerebral pathology and 171 (52,61\%) patients with spinal pathology. Most common surgical procedures performed were: craniotomies for tumours and hematoma removal, minimal invasive procedures for spine, endovascular and vertebroplasty. The median length of stay for emergency patients was significantly longer than that of elective patients ( 13 vs. 8 days). For 215 (66.15\%) patients general anaesthesia was performed, local anaesthesia in 97 (29.84\%) patients, 13 patients (4\%) were not operated. Good quality of life results appreciated by patients and relatives were recorded in 236 cases $(72.61 \%)$ in the first and second category; less better results to those over 85 years old; same symptoms especially pain 63 patients (19.38\%), complications to 47 patients (14,46\%) especially cardiac, renal and

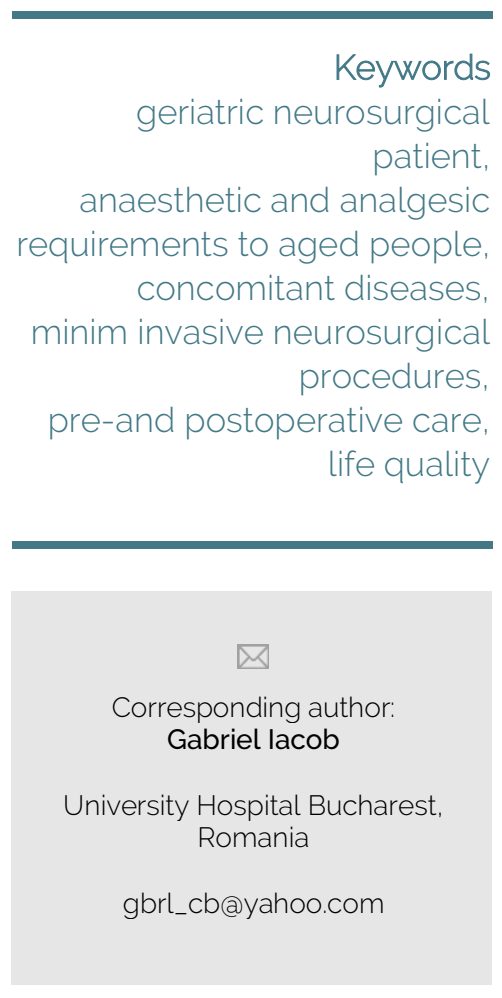

Copyright and usage. This is an Open Access article, distributed under the terms of the Creative Commons Attribution Non-Commercial No Derivatives License (https://creativecommons org/licenses/by-nc-nd/4.0/) which permits noncommercial re-use, distribution, and reproduction in any medium, provided the original work is unaltered and is properly cited.

The written permission of the Romanian Society of Neurosurgery must be obtained for commercial re-use or in order to create a derivative work.

ISSN online 2344-4959

(c) Romanian Society of Neurosurgery

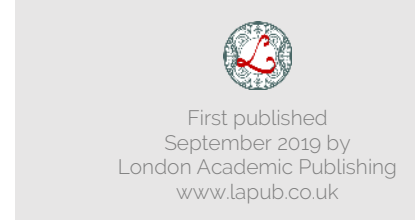


respiratory failures, also motor deficits, seizures, CSF fistula, mortality in 26 cases (8\%), re-admissions in 45 cases (13.84\%) less than 1 month after discharge.

Conclusions. Old prejudices that old age is a contraindication for surgery have to be removed. Clinical and surgical decisions for neurosurgical procedures in the elderly are decisive for limiting reported morbidity and mortality rates. For life quality, realistic family and society expectations, several aspects should be considered for safe and effective results: careful patient selection on patient status, comorbidities and physiological reserve; neurosurgical pathology, urgency of the surgical procedure, the strategy of neurosurgical management based on advances in imaging and interventional radiology, minimal invasive neurosurgical procedures with significant preoperative and postoperative care. Good results could be obtained even in elderly people for chronic subdural hematoma, simple brain or spinal tumour, good grade aneurysm, trigeminal pain, vertebroplasty in spinal vertebral fractures, etc.

\section{INTRODUCTION}

Aging means concomitant a physiological process but also structural degeneration, a graded loss of functional capacity of all organs and tissues (1)(2). The number of elderly patients is increasing worldwide, generating a larger concern (3-7), an increasing problem regarding socio-economic reasons related to the medical act, morbidity and additional care requirements, perioperative adverse events (8-10). It is estimated that between 2000 and 2050 , the proportion of the world's population over 60 years will double, and the number of people aged 80 and older will quadruple (7)(11). For neurosurgical patient, beyond the age of 65 years, both chronic or in emergency, all measures should be institute to maintain quality of life, to avoid perioperative mortality and morbidity (10). In Romania the share of people over 65 years of age, was $18 \%$ of the total population on January 1, 2018, respectively $3,559,957$ persons out of $19,523,621$. According to the NIS data, among the elderly, the men numbered $1,433,412$ persons ( $15 \%$ of the total number of men in Romania of 9,543,228 persons) and the women $2,117,495$ (21\% of the total number of women residing in Romania of 9,980,393 persons)(12).

\section{MATERIAL AND METHOD}

This retrospective study was performed on 325 patients aged 65 years or older, with neurosurgical diseases, admitted to the Neurosurgery or the Intensive Care Unit of our hospital, between 20142019. An analysis was made on variables such as: age, pathology, the admission Glasgow Coma Scale (GCS) score, both chronic or in emergency, comorbidities - using Charlson Comorbidity Index (13)(14), length of hospital stay especially in the ICU unit, type of cranio-cerebral or spinal procedures performed, anaesthesia protocols, complications, performance status, re-admissions morbidity and mortality. $45.8 \%$ of patients had neurosurgical cranial pathology and $54,2 \%$ had neurosurgical spinal diseases. Patients age were divided into three categories: between 65-70 years old there were 152 patients $(46,76 \%)$, between $70-85$ years old 93 patients $(28.61 \%)$ and over 85 years old 80 patients (24,61\%). 173 patients were females (53,23\%), 152 were males (46.76 \%). The admission Glasgow Coma Scale (GCS) score was between 3-15, worse: between 3-5 especially to those over 85 years old in 29 cases $(8.02 \%)$ with early death in 13 patients. A CGS score between 7-14 was recorded to 89 patients (27,38\%): 12 cases $(3.69 \%)$ in the first category, 42 cases (12.95\%) cases in the second and 35 cases $(10.76 \%)$ in the third. Several combined comorbidities were encountered in 294 patients (90.15\%), limiting surgical attitude, also obtaining the informed consent for surgery: cardiac insufficiency, several cardiac diseases with damage to the ejection fraction (myocardial infarction, arrhythmias, valvular stenosis or insufficiency, untreated hypertension); chronic pulmonary disease, bronchial asthma; previous strokes with hemiplegia; severe haematological diseases (lymphoma, leukemia, myeloma, coagulation disorders with thrombocytopenia or coumarinic overdose); hepatopathy (cirrhosis, portal hypertension with variceal bleeding history, chronic hepatitis); kidney failure - patients submitted in the dialysis program; peptic ulcer disease; connective tissue disease; peripheral vascular disease (intermittent claudication, chronic arterial insufficiency with by-pass, untreated thoracic or abdominal aneurysm), psychiatric illnesses (dementia); concomitant systemic disease or immunosuppressed patients by diabetes, primitive and metastatic cancers affecting various organs, chemotherapies, transplant patients, infectious diseases even AIDS; severe osteoporosis, chronic ethylic intoxication, drug allergies.

Neurosurgical pathologies varied alone or concurrently: 154 (47,38\%) patients with cerebral pathology and 171 (52,61\%) patients with spinal pathology: cranio-cerebral and spinal trauma 
(hematomas: epidural, subdural, intracerebral, posttraumatic subarachnoid haemorrhage, cerebral and spinal contusions, DAl, cerebral lacerations, CSF fistula, Schneider syndrome, hematomyelia); cerebral tumours: extraaxial meningioma, metastasis, gliomas especially glioblastoma, schwanomas, pituitary and spinal tumours: extradural, intradural, intramedullary, frequent metastasis in lung, breast, prostate cancer; cerebral aneurysm, carotid-cavernous fistula, MAV, spinal degenerative myeloradiculopathy, mielopathy with cervical and lumbar canal stenosis, spinal instability with osteoporotic vertebral changes, vertebral fractures; Arnold neuralgia, trigeminal, atypical facial pain, spinal neuropathic pain; tremor (Parkinson's syndroms \& disease, dystonic movements), blepharospasm even Meige syndrome, spasmodic torticollis. All patients were carefully neurologic, biologic, neuro-imagistic, multidisciplinary evaluated; neurosurgical surgical indication, anaesthesia protocol, informed consent to be used to each patient were supported with accuracy. Several procedures have been performed: craniotomies for tumours and hematoma removal, minimal invasive procedures for spine, endovascular, vertebroplasty, nerve block, neurovascular decompression or Gamma-Knife for pain procedures have been performed.

\section{RESULTS}

The median length of stay for emergency patients was significantly longer than that of elective patients ranged between 13 vs. 8 days, and in the ICU unit vary from 1 to 63 days.

For 215 (66.15\%) patients general anaesthesia was performed, local anaesthesia in 97 (29.84\%) patients, 13 patients (4\%) were not operated. Good quality of life results appreciated by patients and relatives were recorded in 236 cases (72.61\%) in the first and second category; less better results to those over 85 years old; same symptoms especially pain 63 patients $(19.38 \%)$, complications to 47 patients $(14,46 \%)$ especially cardiac, renal and respiratory failures, also motor deficits, seizures, CSF fistula, mortality in 26 cases (8\%), re-admissions in 45 cases (13.84\%) less than 1 month after discharge. For old patients physical therapists may improve strength and balance, safely walk ability, climb stairs before being released from the hospital. Occupational therapists may help such patients to be able to do personal hygiene using the bathroom, to get dressed; speech language pathologists could help patients with speech, language or thinking.

\section{DISCUSSION}

The elderly patient - defined as an individual 65 years of age and over, still represent all around world in general, a fragile population, a great concern, with higher costs of health care, a difficult issue for the health systems struggling with limited resources (37)(9)(10). For each aging person there are a personal rate of degeneration of CNS as like as the whole body, in anatomy, in mental and physical activity, also in higher costs of living, lifestyle change, loneliness, possible dependence of specific medication for medical associated illnesses - such as diabetes and cardiovascular disease (3)(6). It's also a reality that the elderly population represent the fastest growing segment of the world's population; the world's population over 60 years will double between 2000 and 2050 and the number of people aged 80 and older will quadruple (7). 47,8 million the number of people age 65 and older in the United States on July 1,2015; this group accounted for 14,9 per cent of the total population (15). 98,2 million - the projected population of people age 65 and older in 2060; of this number 9,7 million will be age 85 or older (16). Such growing proportion affect also the neurosurgical admissions both in emergency surgeries or classical presentations for every group of persons on age "young-old" - 65-74, "middle-old" 75-84 and "old-old" > 85 (17). There are several changes involved in different degree with age, with neurosurgical interest too (1)(2)(18-23):

-cerebral atrophy affecting especially the frontal and temporal lobes, decline in brain weight, increase in ventricular size, thickening of meninges, decrease in width of gyri, deep sulci, cortical neurons, myelinated axons, the number of synapses per neuron, loss of Nissl substance, nuclear atrophy; intracellular deposits, granulation or fragmentation of mitochondria. These aspects are generating tolerance in front of any expansive process, clinical onset delay in case of tumours, intracranial hematoma, facilitates surgical approach, allows intracranial expansive processes removal.

-cerebral fragility with decreased cerebral compliance that induces the brain's difficulty to resume its 
anterior position, able to generate hematoma recurrence, hydrocephalus, etc.

-decreased cerebral blood flow, loss of vascular autoregulation and responsiveness to neuronal demand, hypercapnia, heterogeneous regional variations with decreased cellular oxygen, glucose and oxidative metabolism, altered sodium, potassium and calcium homeostasis with reduced axoplasmatic transport, calcium mediated and synaptic neurotransmitter release. There are also general decrease in excitability, peripheral nerve conduction velocity, altered reflex responses and increased latency of evoked potential, a disorganization of highly coordinated activities, with autonomic and homeostatic changes: reduced temperature regulation, orthostatic hypotension, chronic constipation, slowed hear rate, decreased blood pressure.

-leukoaraiosis (gr."leuko-" = white, meaning white matter and the adjective «araios" ="thin."- Hachinski V. 1987) are typically generated by: lacunar stroke/transient ischemic attack (TIA), dementia of both vascular and non-vascular etiologies (hypertension, degenerative, in Alzheimer's disease), sporadic cerebral amyloid angiopathy, diabetes; the precise pathogenic mechanisms remain unclear. Recent genetic results strongly supported that leukoaraiosis is associated with immune response and neuroinflammation (23). Pathologically, leukoaraiosis is characterized by white matter gliosis, axonal and myelin loss, increased perivascular spaces, patchy demyelination, hyaline thickening and arteriosclerosis of small vessels that can evolve to lipohyalinosis, fibrinoid necrosis, and denudation of ependyma. Main clinical manifestations are cognitive and executive troubles (memory loss especially short-term memory, visual and hearing loss, declining endurance, verbal intelligence, processing speech dysfunction in learning and language skills, depression), ankle jerks decreased or absent, increased primitive reflexes (glabella, palmomental), slowed forward flexed, altered gait, tremor with loss of fine motor coordination bladder instability. There are hypo-dense areas to cerebral CT scan, diffuse, heterogeneous, imprecisely delimited, primarily interesting the centre of the white substance or the immediate subcortical areas. The MRI examination in weighted $T_{1}$ sequences shows hypointense areas and the weighted $T_{2}$ sequences show hyperintense areas; sometimes without correlation as number, localization. MRI findings are commonly seen to elderly people with prevalence ranging from $50 \%$ to $100 \%$. Leukoaraiosis may explain poor clinical outcomes and increases the risk of disability, dementia, depression, stroke, and the overall morbidity and mortality.

-amyloid angiopathy > 70 years: explains the difficulties of hemostasis, haemorrhagic recurrences, preanesthetic assessment, informed consent, perioperative evaluation, risk issues especially for those people identified with ASA physical status of III or IV. and care are magnified in older patient, anaesthetic management, efficient surgical skills procedures - especially functional neurosurgery, radiosurgery, planned postoperative management may contribute to successful outcomes also to ameliorate quality of life related to neurosurgical diseases, also to a specific neurosurgical pathology, especially pain (3)(5)(7)(8)(10)(11)(24-29). There are medical factors affecting anaesthesia: multiple medical comorbidities including sepsis, metabolic disturbances severe obesity or poor nutritional status, alcohol and/or drug abuse, history of cardiac failure or myocardial infarction $\leq 1$ year with low ejection fraction (EF) on echocardiography, severe uncontrolled hypertension, severe respiratory dysfunction, hepato-renal failures, neurovascular, psychiatric disease, history of CVA or TIA, peripheral vascular disease, immuno-compromised patients by diabetes, tuberculosis, etc; severe coagulopathy status with trombocytemia, haemophilia, antiplatelet agents even help syndrome (to old patients even a normal coagulogram may coexist with difficult hemostasis), cognitive function at risk especially at advanced age $>70$ years: cognitive impairment, acute confusional state, delirium. There are also surgical factors affecting anaesthesia in all such high-risk geriatric patients which should be discussed in detail for optimizing the outcome with the anaesthesiologist and cardiologist (28)(29):

-surgical position: prone, sitting, Concorde position have always cardio-respiratory implications, Wilson Frame or similar with varying degrees of inclination, neck stabilization, spine traction, requirement of elective ventilation, head up to 10 degree and reverse Trendelenburg may avoid perioperative blindness by direct/indirect eye pressure, ischemic 
optic neuropathy, central retinal artery occlusion (CRAO), cortical blindness. Also a severe spinal trauma or an important scoliosis, osteoporosis, osteomyelitis, severe obesity or undernourished people.

-plan of surgical excision, anticipated duration (in general surgery should never exceed $6.5 \mathrm{~h}$ in older patient), anticipated blood loss, neurophysiologic monitoring, postoperative ventilation. The anaesthetic regimen has to be tailored according to the physiological reserve: local or general anaesthesia. For general anaesthesia there are several remarks:

-before induction the invasive monitoring for arterial blood pressure - ABP and central venous pressure CVP should be instituted under local anaesthesia.

-induction should be performed with fentanyl, etomidate (etomidate requirement beyond the age of 80 years may decrease by $50 \%$,), non depolarisant muscular blockade agents intravenously. Propofol produces an exaggerated fall in blood pressure especially to old patients, dehydrated, midazolam has increased duration and potency, neuromuscular blocking agents are unchanged oral intubation.

-anaesthesia should be maintained with $50 \%$ oxygen, air, sevoflurane in minimum alveolar concentration MAC of 0.5 (MAC requirement for volatiles also decreases in the elderly, though the onset may be delayed due to decreased cardiac output). Monitoring should be performed by pulse oximetry: $\mathrm{SpO}_{2}$, arterial blood pressure ABP avoiding acidosis, hyper or hypotension - $84 \mathrm{~mm}$ systolic minimum, Hct $>28 \% /, \mathrm{Hb}>9.4$ with cardiac index (stroke volume) $\downarrow$ 24\%; blood loss > 45\%, heart rate HR, electrocardiogram ECG, end-tidal carbon dioxide, hourly urine output, temperature. To maintain hemodynamics and to avoid cerebral perfusion pressure crash crystalloids and noradrenaline should be infused intraoperatively.

-even in case with normal coagulation test, haemostasis could be laborious in the confined space of the cranial cavity or the spinal canal presenting as the surgical field; in case of bleeding diathesis (due to prescribed medications in the form of clopidogrel, warfarin, etc., alone or more often in combination) especially in emergency this is dangerous. For such situations there are several costly, time consuming, delaying procedures: red blood cell transfusion, donor platelet, frozen plasma, Novoseven, Pronative, Hemocompletan, recombinant factor VIla, all of this are costly, time consuming, delay procedures.

-postop. Slowly awakening, extubated and closely watched in neurosurgical intensive care unit to improve resource utilization, decreased in-hospital mortality (26). The effect of depth of anaesthesia on outcomes is still a grey area. Perioperative cardiac maintaining volume status, pulmonary evaluation are reliable predictors of complications, (ex. unoptimized pulmonary diseases, increased closing volumes and decreased expiratory flow rates, tracheostomy, postoperative mechanical ventilation) predispose older patients to complications and death, also renal status avoiding nephrotoxic drugs, prevention of hyperglycemia or hypoglycemia, adequate prophylaxis for deep venous thrombosis even with pneumatic compression devices, multiple medications for other co-morbidities. Mental status should be assessed preoperatively because dementia is a predictor of poor outcome perioperatively, increasing mortality by $50 \%$ (25). Nutritional deficiencies should be corrected because they play a role in wound healing and recovery albumin levels of $<3.2 \mathrm{~g} / \mathrm{dl}$ in hospitalized elderly patients increased perioperative mortality (28), also pain should be treated.

Most common geriatric pathology are:

-traumatic brain injuries, more common: subdural uni or bilateral hematoma - a common pathology (11)(30-35), mainly affecting elderly patients, " not a benign disease" (34) In patients over 80 years old chronic subdural hematoma incidence is reported $127.1 / 100.000 / y e a r$, with a mortality of less than $10 \%$, a history of head injury is not always admitted, several cases could be under anticoagulant treatment because of cardiac pathologies or ictus cerebri, arterial hypertension, diabetes mellitus with severe thrombocytopenia, high INR, even with a normal coagulogramm haemostasis may be difficult. The most common clinical presentation include hemiparesis, disphasia/ aphasia, disorientation, hemianopsia. Diagnosis is sustained on CT scan. Anaesthesia should be local or general, to correct a severe coagulopathy may be necessary: thrombocytes, Novoseven-factor VII, Pronativ, Hemocompletan in case of fibrinolysis. Several 
surgical techniques could be used: craniotomy centred on the thickest portion of the clot (useful in clots that can't be evacuated through a simple trepan hole, allows brain to resume its anterior position, to evacuate a concomitant intracerebral hematoma, to monitor intracranial pressure in those with CGS $<9$ Bullock et al, allows haemostasis), craniotomy, useful when intracranial pressure is maintained, requiring removal of the bone, trephination or two - several trepan holes in the thickest parts of the hematoma (for example, frontal and parietal that may be needed to be incorporated into a fronto-temporoparietal craniotomy in case of relapse, hematoma consistency, thick membranes), the subdural evacuating port system (SEPS): the minimally invasive technique, a safe method, effective in subacute and chronic subdural hematoma in elderly patients, easy to perform with local or general anaesthesia, it takes less than 10 minutes, without irrigation, aspiration, catheter (36). Postop complications are: recurrences, persistence of preop. brain position by decreased cerebral compliance, parenchymal haemorrhages in the same hemisphere or controlateral. Outcome are measured with Glasgow outcome scale (GOS) or Markwalder grading system (MGS) to evaluate improvement in neurological function in general a good GOS in $86.1 \%$ of patients older than 80 years (11), the discharge disposition to return to an independent life, to rehabilitation or nursing home. There are also cerebral contusions and lacerations, rare extradural hematoma with late shift effect of cerebral parenchima, veins ruptures and haemorrhagic events, dimished recovery capacity.

-cerebral aneurysm and less frequent cerebral arteriovenos malformation with subarahnoidian haemorrhage, ischemic \& haemorrhagic strokes supra and infratentorial, associated with hydrocephalus and occlusive cerebrovascular disease 40\% (3)(37).

-epilepsy to older patients is are more frequent (the annual incidence is 85.9 per 100,000 for people aged 65-69 years and 135 per 100,000 for those aged over 80 years (38); similar status epilepticus appears to occur more frequently with significantly greater morbidity and mortality in this age group (39). There are focal seizures, automatisms without generalisation or with secondary generalisation, the postictal phase is prolonged with confusion and memory problems corresponding with focal changes on electroencephalograph (EEG) and neuroimaging epileptogenic lesion (40). Epilepsy are more common encountered with stroke - the risk of epilepsy increases up to 20-fold in the first year after a stroke, hypertensive encephalopathy, cerebral vasculitis, tumours (gliomas, meningiomas and metastases), trauma, dementia, Alzheimer's disease - are up to 10 times more likely to develop epilepsy than those without the condition (38). To older patients there are: side-effects, toxicity, increased susceptibility to anti-epileptic drug, a mortality rate 2-3 times higher than the general population, a very variable prognosis depending on the epilepsy syndrome, the frequency of seizures, the response to treatment - an inadequate seizure control raise the suspicion of poor adherence or progressive neurodegenerative disease; social difficulties, multidisciplinary service requirements in the community (41).

-cerebral tumours:

meningiomas: are $12.8 / 100,000$ incidence to those of $\geq 65$ years of age, increasing with age (42)

In the elderly, meningiomas are diagnosed small, slow growing, asymptomatic extraaxial tumours without brain edema, even calcified, occurring more frequently in women than men (43). For such tumours therapeutic attitude are conservative clinical observation and radiologic follow-up (44-46). For clinically symptomatic and fast growing meningioma, especially atypical or malignant, to elderly patients with limited physiological capacities and comorbidities, surgery after a careful consideration should be the mainstay of treatment for local tumour control (47-52), to realise genetic and histological diagnosis (it's possible to the same patient to see meningiomatosis with different genetic and histologic behaviour: benign and malignant meningiomas), but also stereotactic radiotherapy (SRT), Gamma knife, Linac-based stereotactic radiosurgery effective in terms of tumour control and survival, safe with regard to toxicity - the overall rates of complications ranged from $2.7 \%$ to $29.8 \%$, and the overall incidence of complications 20\% (range, 3-61\%)(53-59).

Metastasis are increasing to the majority of cancer patients over the age of 65 , not only cerebral or intradural spinal metastasis, but more frequent bone metastasis, involving the spine in approx. 50\% (60). Elderly survivors of breast cancer, lung cancer, and melanoma face risk of brain metastasis later in life; rates of synchronous brain metastases incidence 
proportions in lung, breast, and melanoma cancers were $9.6 \%, 0.3 \%$, and $1.1 \%$, respectively; such tumours may require extra surveillance in the years following initial cancer treatment. Non-small cell lung cancer made up the majority of lung cancer SBM IP, at $13.4 \%$, and small-cell lung cancer made up the majority of lung cancer - lifetime brain metastases at $23.1 \%$. The most frequent spinal metastases $(60 \%)$ are from breast, lung, or prostate cancer (61). The chance that an elderly patient (60-79 years old) is affected by bony metastases is four times higher in men and three times higher in women than a middle-aged patient (40-59 years old). Pain, neural compression with motor deficits, pathological fracture, and instability are the most common surgery indication. For cervical area: the occipitocervical junction can generally be treated by posterior resection and stabilization, in the middle and lower cervical spine the anterior approach with anterior decompression and anterior column reconstruction; in the thoraco-lumbar spine a posterior decompression and postero-lateral vertebral body resection through a posterior approach only, with a concomitant reconstruction and stabilization. There are also for paleative cases different treatment modalities-irradiation, chemotherapy, steroids, biphosphonates, morphine pump. Since prospective randomized studies comparing different treatment modalities for spinal metastases including surgery are not available and are ethically difficult to achieve, each case remains an interdisciplinary, shared decision-making process for what is considered best for a patient or elderly patient. However, whenever surgery is an option, it should be planned before irradiation since surgery after irradiation has a significant higher complication rate.

Glioblastoma multiforme are at old age a high incidence, also most significant associated with poor prognosis, even after the introduction of temozolomide (TMZ) in 2006, without any clear borderline (62). After published statistics from the Japan Brain Tumour Registry from 2001 to 2004 (63), patients aged $>65$ years and $>75$ years accounting for $42 \%$ and $11.4 \%$, respectively; the most frequent age group of patients was 65-69 years, which accounted for $17 \%$ of the cases, median overall survival was 15 months. GBMs are subdivided according to their molecular phenotype see WHO classification of central nervous system (CNS) tumour 2016 (64), namely, CpG island methylator phenotype (G-CIMP) and isocitrate dehydrogenase (IDH) mutation: IDH wild type and IDH mutant absence of IDH mutation, is the most predominant GBM in the elderly. Also MGMT promoter methylation is independent of age, with $47 \%$ in the elderly, epidermal growth factor receptor EGFR, p53, CDKN2A, PTEN were not prognostic factors (65), vascular endothelial growth factor (VEGF) was higher in GBM in the elderly than in young patients, also the prognostic value of telomerase reverse transcriptase (TERT) promoter mutation in GBM has been debated (66). Although age is a poor prognostic factor, old age alone is suggested to have no association with poor prognosis, an aggressive treatment should not be withheld because of old age (67). Multimodal treatment in elderly patients with GBM aged $>65$ years should include:

-surgical resection: achieving maximal tumour cytoreduction within the safety margin, brain decompression, histopathological and genetic diagnosis especially for $I D H$ mutation and MGMT methylation status. Such aggressive attitude may prolonge survival by 2.8 times than biopsy (median OS: 171 days after the craniotomy versus 85 days after the biopsy), also for aged patients $\geq 75$ years may extend survival by 2 months, delayed tumour progression and improved functional prognosis (68-76).

-radiotherapy - hypofractionated radiotherapy (Hypo-RT $40 \mathrm{~Gy} / 15 \mathrm{Fr}$ ) alone can be considered if the tumour has an unmethylated MGMT promoter (7780)

-with concurrent and adjuvant TMZ - in elderly patients with MGMT methylated tumours monotherapy with TMZ can expect prolonged survival (78-82); clinical benefits of bevacizumab use in remain unclear (83)(84), also lack of evidence regarding the efficacy of carmustine wafer (85)(86).

another cerebral tumor: schwanoma, pituitary adenoma (87)

-spinal pathology means for old patients a wide range of neurosurgical procedures to solve:

spinal degenerative myeloradiculopathy, mielopathy with cervical canal stenosis, lumbar spinal stenosis (it's important to clarify that surgery will not give 
another spine, also surgery is better to be done in a only surgical procedure); spinal tumours: extra/intra dural, intramedullary and more frequent vertebral metastasis in lung, brest or prostate cancers; spinal instability and osteoporotic vertebral changes.

-chronic geriatric pain defined as "an unpleasant sensory and emotional experience associated with actual or potential tissue damage", is a longstanding pain that persists beyond the usual recovery period or occurs along with a chronic health condition, for greater than 3 months is a more frequent neuropathic, persistent, recurrent, underreported; much more difficult to treat especially for those with long-lasting pain compared to young people, justified by pain corticalisation (88). Such condition is impairing activities of daily living, ambulation, quality of life; more difficult to be understood especially to those patients with cognitive or language impairments; also may generate polypharmacy with concomitant medication, but also responsive for poor health, accidents, gait abnormalities, cognitive decline, denial, depression, psychosocial concerns, with direct and indirect estimated treatments costs close to $\$ 50$ billion a year (89). There are several types of chronic pain, complex, multifactorial in older population, very debilitating and painful: trigeminal neuralgia, atypical facial pain, occipital neuralgia, postherpetic neuralgia, headache, stroke and phantom limb pain, failed spinal surgery, referred or pain related to cancer; also pain perception is more difficult to be treated. For such patients a multidisciplinary approach needs to be conceived: pharmacotherapy, psychological support, psychological support, also interventional procedures. A wide range of procedures (88) should be wisely proposed taking into account life expectancy, comorbidities, the patient's desire focusing on neuromodular rather than lesional techniques, also risks for each procedure: microvascular decompression, chemical neurolysis, radiofrequency and glycerol rhizotomy, stereotactic radiation, deep brain \& spinal cord stimulation, motor cortex stimulation and neuraxial drug delivery.

-tremor (Parkinson's syndrome \& disease and dystonic movements) treated especially by medication, DBS, motor cortex stimulation (90).

-neuro-infections especially to immunocompromise patients: diabetes, TB, HIV, etc.

-multiple sclerosis

-dementia

\section{CONCLUSIONS}

This study is a plea argue that in elderly patients, which is likely to continue to grow (6), risk factors should be adequately managed even in emergency (3), with correct preoperative evaluation, appropriate perioperative care (8), titrated anesthetic management, efficient surgical skills. Planned postoperative management may improve neurological status (30)(91), outcomes, reduce mortality rates, reduce length of intensive care stay, improved resource utilisation, fiscal benefits (7)(9). Further studies in the elderly are mandatory to be performed to improve clinical decisions on risk benefit ratios pending on new technologies.

\section{REFERENCES}

1. Hickey R., Sloan T.B. - Physiologic Changes with Aging in the Central Nervous System, in Albin M.S. Text book of Neuroanesthesia with Nerosrgical and Neuroscience Perspective, 1997, 37, 1349-1360.

2. Arseni C., Balaceanu Stolnici C., Nica I. Neurogeriatrie, Editura Medicala 1984.

3. Dujovny M, Charbel F - Geriatric neurosurgery, Surg Neurol. 1987, 28(1), 10-16.

4. O'Brien D.P, Nagaria J., et al. - Neurosurgery for the Elderly: Facts and Figures, Gerontology 1996, 42, 16.

5. Rha H.K., Lee K.J. et al. - Neurosurgery for the Elderly, Journal of Korean Neurosurgical Society 1997, 26 (12), 1699-1704.

6. Chibbaro S, Di Rocco F et al. - Neurosurgery and elderly: analysis through the years, Neurosurg Rev. 2010, 34(2), 229-234.

7. González-Bonet LG, Tarazona-Santabalbina FJ, et al. - Neurosurgery in the elderly patient: Geriatric neurosurgery, Neurocirugia (Astur). 2016, 27(4), 155-166.

8. Jin F, Chung F. - Minimizing perioperative adverse events in the elderly. Br J Anaesth 2001, 87, 608-24.

9. McGory ML, Kao KK, et al. - Developing quality indicators for elderly surgical patients. Ann Surg 2009, 250, 338-347.

10. Bhatnagar V., Datta S.G.S., et al. - The perioperative concerns of emergency neurosurgery in elderly patients: A series of three cases, Journal of Marine Medical Society 2017, 9, 1, 58-60.

11. Gelabert-González M, Fernández-Villa JM, et al. Chronic subdural hematoma in patients over 80 years of age, Neurocirugia (Astur). 2001, 12, 325-330. 
12. INS - Romania, Ponderea persoanelor varstnice, de peste 65 de ani, Mediafax, 30 Septembrie 2018, ora 14:19.

13. Charlson ME, Pompei P, et al. - A new method of classifying prognostic comorbidity in longitudinal studies: development and validation. J Chronic Dis 1987;40:373-83.

14. Zhang JX, Iwashyna TJ, et al. - The performance of different lookback periods and sources of information for Charlson comorbidity adjustment in Medicare claims, Med Care 1999; 37,1128-39.

15. United States Census Bureau -Vintage 2015 Population Estimates.

16. United States Census Bureau - 2014 National Population Projections.

17. Leppik I - Antiepileptic drug trials in the elderly. Epilepsy Res. 2006 Jan, 68(1), 45-48.

18. Hickey R., Sloan T.B. - Physiologic Changes with Aging in the Central Nervous System, in Albin M.S. Text book of Neuroanesthesia with Neurosurgical and Neuroscience Perspective,1997, 37, 1349-1360.

19. Duckett S. - The normal aging human brain, in Duckett S (ed): The Pathology of the Aging Human Nervous System, Philadelphia, Lea \&Febiger 1991, 1-19.

20. Morris J.C. McManus D.Q. - The neurology of aging: Normal versus pathologic change, Geriatrics 1991, 46-47.

21. Vital C, Vital A. - Peripheral neuropathy, in Duckett $\mathrm{S}$ (ed): The Pathology of the Aging Human Nervous System, Philadelphia, Lea \& Febiger 1991, 393-432.

22. Berhouma M., Salmon P.K.- Brain and Spine Surgery in the Elderly, Neurosurg Rev. 2010, 34 (2), 229-234.

23. Qing Lin, Wen-Quing Huang et al. - Genetic association of leukoaraiosis indicate pathophysiological mechanisms in white matter lesions etiology, Reviews in the Neurosciences, 2015-03_11, vol 26, issue 3.

24. Audisio RA, Ramesh H, et al. - Preoperative assessment of surgical risk in oncogeriatric patients. Oncologist 2005, 10, 262-268.

25. Cruickshank G.H, Marshall .I - Neuroanesthesia in the elderly, Current Anesthesia \&Critical Care 1997, 8,3, 103-107.

26. Lang JM, Meixensberger J, et al. - Neurosurgical intensive care unit-essential for good outcomes in neurosurgery? Langenbecks Arch Surg. 2011, 396(4), 447-451.

27. Maurice-Williams RS, Kitchen N. - The scope of neurosurgery for elderly people, Age Ageing. 1993, 22(5), 337-342.

28. Ciurea A.V., Iacob G. - Technici neurochirurgicale, Cartea Universitara 2006, 1-28.

29. Wong D. - Recognizing and Controlling PreOperative Anesthesia and Surgical Risk Factors in the Older Patient, Dubai Neurosurgery Congress 2015.
30.Zoia C, Verlotta M, et al. - Chronic Subdural Haematoma in Patients Over 90 Years: Outcome after Surgical Treatment, Austin Neurosurg Open Access. 2014, 1(5), 1022.

31. Lee L, Ker J, Ng HY et al. - Outcomes of chronic subdural hematoma drainage in nonagenarians and centenarians: a multicenter study, J Neurosurg. 2016, 124(2), 546-551.

32. Mulligan P, Raore B, Liu S, Olson JJ. Neurological and functional outcomes of subdural hematoma evacuation in patients over 70 years of age, J Neurosci Rural Pract. 2013, 4, 250-256.

33. Stippler M, Ramirez P, et al. - Chronic subdural hematoma patients aged 90 years and older, Neurol Res. 2013, 35, 243-246.

34. Miranda LB, Braxton E, et al. - Chronic subdural hematoma in the elderly: not a benign disease, J Neurosurg. 2011, 114, 72-76.

35. Gelabert-González M, Fernández-Villa JM, et al. Chronic subdural hematoma in patients over 80 years of age, Neurocirugia (Astur). 2001, 12, 325-330.

36. Kenning T.J., Dalfino J.C.. et al. - Analysis of the subdural evacuating port system for the treatment of subacute and chronic subdural haematomas, J.Neurosurg. 2010, 113(5), 1004-1010.

37. Ballotta E, Da Giau G, et al. - Early and long-term outcomes of carotid endarterectomy in the very elderly: An 18-year single-center study. J Vasc Surg 2009, 50, 518-525.

38. Johnston A., Smith P.E - Epilepsy in the elderly, Expert Rev Neurother. 2010, 10(12), 1899-1910.

39. Cloyd J, Hauser W, et al. - Epidemiological and medical aspects of epilepsy in the elderly, Epilepsy Res. 2006 Suppl 1:S39-48.

40. Scottish Intercollegiate Guidelines Network - SIGN 2015: Diagnosis and management of epilepsy in adults.

41. Elger C.E., Schmidt D. - Modern management of epilepsy: a practical approach. Epilepsy Behav. 2008, 12(4), 501-39.

42. Claus E.B., Bondy M.L. et al. - Epidemiology of intracranial meningioma, Neurosurgery 2005, 57, 6, 1088-1095.

43. Lee J, SadeB. - The novel 'CLASS' algorithmic scale for patient selection in meningioma surgery, in Meningiomas, Lee J., Springer London, UK ed.,2009, 217-221.

44. Bateman B.T., Spellman J.P., et al. - Meningioma resection in the elderly: Nation wide in patient sample, 1998-2002, Neurosurgery 2005, 57, 5, 866871.

45. Cahill K. S, Claus E. B. - Treatment and survival of patients with nonmalignant intracranial meningioma: results from the surveillance, epidemiology, and end results program of the National Cancer Institute. 
Clinical article, Journal of Neurosurgery 2011, 115, 2, 259-267.

46. Dolecek T.A., J. M. Propp J.M. et al. - CBTRUS statistical report: primary brain and central nervous system tumors diagnosed in the United States in 2005-2009, Neuro-Oncology 2012, 14, suppl. 5, v1v49.

47. Patil C.G., Veeravagu A. et al. - Craniotomy for resection of meningioma in the elderly: a multicentre, prospective analysis from the national surgical quality improvement program," Journal of Neurology, Neurosurgery and Psychiatry 2010, 81, 5, 502-505.

48. Schul D. B., Wolf S., et al. - Meningioma surgery in the elderly: outcome and validation of 2 proposed grading score systems, Neurosurgery 2012, 70, 3, $555-565$.

49. Maurice-Williams RS, Kitchen ND.- Intracranial tumours in the elderly: the effect of age on the outcome of first time surgery for meningiomas, $\mathrm{Br} \mathrm{J}$ Neurosurg. 1992, 6(2), 131-137.

50. Tucha O., Smely C., Lange K. W., - Effects of surgery on cognitive functioning of elderly patients with intracranial meningioma, British Journal of Neurosurgery, 2001, 15, 2, 184-188.

51. Poon M. T.C., Fung L. H.K., et al. - Outcome of elderly patients undergoing intracranial meningioma resection a systematic review and meta-analysis, British Journal of Neurosurgery 2014, 28, 3, 303309.

52. Boviatsis E. J., Bouras T.I., et al. - Impact of age on complications and outcome in meningioma surgery, Surgical Neurology 2007, 68, 4, 407-411.

53. Iwai Y., Yamanaka K., Ikeda H. - Gamma knife radiosurgery for skull base meningioma: long-term results of low-dose treatment, Journal of Neurosurgery 2008, 109, 5, 804-810.

54. Komotar R.J., Lorgulescu J.B., et al. - The role of radiotherapy following gross-total resection of atypical meningiomas, Journal of Neurosurgery 2012, 117, 4, 679-686.

55. Kaul D., Budach V., et al. - "Linac-based stereotactic radiotherapy and radiosurgery in patients with meningioma," Radiation Oncology, 2014, 9, 1, 78.

56. Kaul D., Budach V. et al. - Outcome of Elderly Patients with Meningioma after Image-Guided Stereotactic Radiotherapy: A Study of 100 Cases, BioMed Research International, 2015, article ID 868401.

57. Fokas E., Henzel M., et al. - Stereotactic radiotherapy of benign meningioma in the elderly: clinical outcome and toxicity in 121 patients, Radiotherapy and Oncology 2014, 111, 3, 457-462.

58. Pechlivanis I., Wawrzyniak S., et al. - Evidence level in the treatment of meningioma with focus on the comparison between surgery versus radiotherapy: a review, Journal of Neurosurgical Sciences 2011, 55, 4, 319-328.

59. Stessin A.M., Schwartz A., et al., - "Does adjuvant external-beam radiotherapy improve outcomes for nonbenign meningiomas? A Surveillance, Epidemiology, and End Results (SEER)-based analysis," Journal of Neurosurgery, 2012, 117, 4, 669-675.

60. Ascha MS, Ostrom QT, et al. - Lifetime occurrence of brain metastases arising from lung, breast, and skin cancers in the elderly: a SEER-Medicare study, Cancer Epidemiol Biomarkers Prev 2019, 28(5), 917-925.

61. Aebi M - Spinal metastasis in the elderly, Eur Spine J. 2003 Oct; 12(Suppl 2), S202-S213.

62. Okada M., Miyake K., Tamiya T. - Glioblastoma Treatment in the Elderly, Neurol Med Chir (Tokyo), 2017, 57(12), 667-676.

63. Committee of Brain Tumor Registry of Japan : Report of the Brain Tumor Registry of Japan (20012004), Neurol Med Chir (Tokyo)2014, 54, 1-102.

64. Louis DN, Ohgaki H, Wiestler OD, et al. WHO Classification of Tumours of the Central Nervous System. WHO/IARC Classification of Tumours, 4th Edition Revised, 2016.

65. Bozdag S, Li A, Riddick G, et al.: Age-specific signatures of glioblastoma at the genomic, genetic, and epigenetic levels. PLoS ONE 8, 2013, e62982.

66. Arita H, Yamasaki K, et al. : A combination of TERT promoter mutation and MGMT methylation status predicts clinically relevant subgroups of newly diagnosed glioblastomas, Acta Neuropathol Commun 2016, 4, 79.

67. Gately L, Collins A, Murphy M, Dowling A: Age alone is not a predictor for survival in glioblastoma. $\mathrm{J}$ Neurooncol 2016, 129, 479-485.

68. Sahebjam S., McNamara Mairead, et al. Management of Glioblastoma in the Elderly Clinical Advances in Hematology \& Oncology 2012, 10, 6, 379-386.

69. Iwamoto FM, Reiner AS, et al. - Patterns of care in elderly glioblastoma patients. Ann Neurol. 2008, 64, 628-634.

70. Yovino S, Grossman SA. - Treatment of glioblastoma in "elderly" patients. Curr.Treat Options Oncol. 2011, 12, 253-262.

71. Ewelt C, Goeppert M, et al. - Glioblastoma multiforme of the elderly: the prognostic effect of resection on survival. J.Neurooncol. 2011, 103, 611618.

72. Chaichana KL, Chaichana KK, et al. - Surgical outcomes for older patients with glioblastoma multiforme: preoperative factors associated with decreased survival. Clinical article. J Neurosurg. 2011, 114, 587-594.

73. Sahebjam S., McNamara Mairead, et al. Management of Glioblastoma in the Elderly Clinical 
Advances in Hematology \& Oncology 2012, 10, 6, 379-386.

74. Oszvald A, Guresir E, et al. - Glioblastoma therapy in the elderly and the importance of the extent of resection regardless of age. J Neurosurg 2012, 1162, 357-364.

75. Noorbakhsh A, Tang JA, et al. : Gross-total resection outcomes in an elderly population with glioblastoma: a SEER-based analysis. J Neurosurg 2014, 120: 3139.

76. Babu R, Komisarow JM, et al. - Glioblastoma in the elderly: the effect of aggressive and modern therapies on survival. J Neurosurg 2016, 124, 998-1007.

77. Roa W, Brasher PM, et al. : Abbreviated course of radiation therapy in older patients with glioblastoma multiforme: a prospective randomized clinical trial. J Clin Oncol 2004, 22: 1583-15880.

78. Cao JQ, Fisher BJ et al - .Hypofractionated radiotherapy with or without concurrent temozolomide in elderly patients with glioblastoma multiforme: a review of ten-year single institutional experience. J Neurooncol. 2012, 107, 395-405.

79. Zarnett OJ, Sahgal A, et al. - Treatment of elderly patients with glioblastoma: a systematic evidencebased analysis, JAMA Neurol 2015, 72, 589-596.

80. Yin AA, Zhang LH, et al. - Radiotherapy plus concurrent or sequential temozolomide for glioblastoma in the elderly: a meta-analysis. PLoS ONE 2013, 8: e74242.

81. Stupp R, Hegi ME, et al. - Effects of radiotherapy with concomitant and adjuvant temozolomide versus radiotherapy alone on survival in glioblastoma in a randomised phase III study: 5-year analysis of the EORTC-NCIC trial. Lancet Oncol. 2009, 10, 459466.

82. Stummer W, Nestler U, et al. - Favorable outcome in the elderly cohort treated by concomitant temozolomide radiochemotherapy in a multicentric phase II safety study of 5-ALA. J Neurooncol. 2011, 103, 361-370.

83. Chinot OL, Wick W, et al. - Bevacizumab plus radiotherapy-temozolomide for newly diagnosed glioblastoma. N Engl J Med 2014, 370: 709-722.

84. Reyes-Botero G, Honnorat J, et al. - Temozolomide plus bevacizumab in elderly patients with newly diagnosed glioblastoma and poor performance status: An Anocef phase II trial. J Clin Oncol 2013, 30: abstr 2020.

85. Westphal M, Ram Z, et al. - Executive Committee of the Gliadel Study Group : Gliadel wafer in initial surgery for malignant glioma: long-term follow-up of a multicenter controlled trial. Acta Neurochir (Wien) 2006, 148: 269-275; discussion 275.

86. Chaichana KL, Zaidi H, et al. - The efficacy of carmustine wafers for older patients with glioblastoma multiforme: prolonging survival. Neurol Res 2011, 33: 759-764.

87. Locatelli M., Bertani G., et al. - The trans-sphenoidal resection of pituitary adenomas in elderly patients and surgical risk, Pituitary 2013, 16, 2, 146-151.

88. Ciurea A.V., Iacob G. - Technici neurochirurgicale, Cartea Universitara 2006, 104-214.

89. American Geriatrics Society Panel on Pharmacological Management of Persistent Pain in Older Persons. Pharmacological management of persistent pain in older persons. J Am Geriatr Soc. 2009, 57(8), 1331-1334.

90. Ciurea A.V., Iacob G. - Technici neurochirurgicale, Cartea Universitara 2006, 216-294.

91. Whitehouse KJ, Jeyaretna DS, et al. - Neurosurgical Care in the Elderly: Increasing Demands Necessitate Future Healthcare Planning, World Neurosurg. 2016, 87, 446-454. 